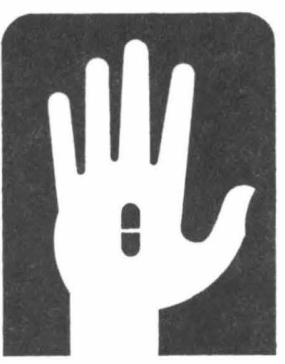

\title{
VITAMIN AND MINERAL SUPPLEMENTS
}

\author{
Charlotte A. Pratt, Ph.D., R.D.
}

Extension Specialist, Nutrition and Foods

Do you take any supplements "just to be sure"? "To give you energy"? or "To make up for what is not in food"? Advertisements on radio and television and in magazines and newspapers constantly warn us that we may not be getting enough vitamins and minerals in our food. Colorful bottles in healthfood stores, pharmacies, and supermarkets remind us that vitamin and mineral supplements guarantee good health. Nearly one-third to one-half of the population consumes one or more vitamin and mineral supplements daily. A recent survey in seven states found $60 \%$ of consumers ingesting from one to 20 supplements daily. The nutrient supplement business not only costs consumers about two million dollars yearly, but also provokes fundamental questions concerning necessity, cost, and danger to health.

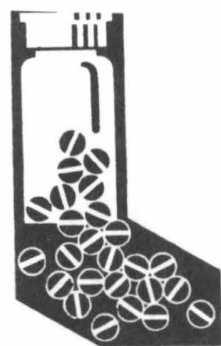

Vitamin and Mineral Toxicities

The slogan "more of a good thing is better" is not necessarily true for vitamin and mineral supplements, especially for the fat-soluble vitamins. Fat-soluble vitamins are stored in the body and they are harmful when consumed in large quantities. Various food faddists have claimed that megadoses (more than 10 times the recommended dietary allowance or RDA) of water-soluble vitamins are not harmful because the excess is excreted in the urine. These faddists also claim that megadoses of certain vitamins prevent cancer, the common cold, and mental disorders. Research has shown that high doses of water-soluble vitamins may indeed be toxic. The mechanisms by which toxicity could occur are: 1) the vitamin or the breakdown products of the vitamin may be directly toxic; 2) large doses of water-soluble vitamins may lead to dependency states and withdrawal symptoms may develop once the vitamin use is discontinued; 3) megadoses of vitamins may mask the symptoms of concurrent disease; and 4) megadoses of vitamins may interfere with the absorption and utilization of drugs or other vitamins. Table one lists some of the symptoms of vitamin and mineral overconsumption. 
A762

no. $348-372$

$\checkmark P$ I

spec

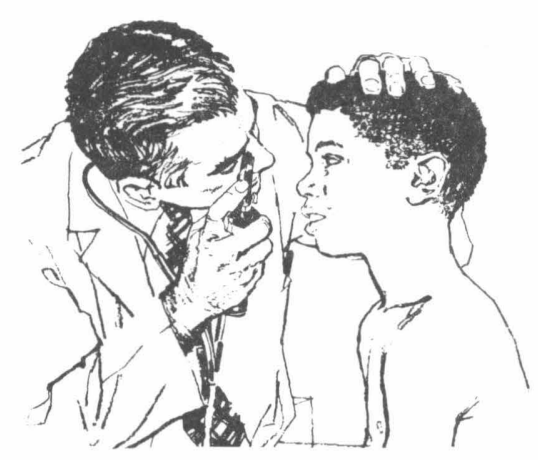

TABLE 1

Vitamin or Mineral

Symptoms of toxicity

Vitamin $\mathrm{C}$

Diarrhea, rebound scurvy, high blood uric acid, false positive urine test for diabetes

Nicotinic Acid

Elushing, diarrhea, nausea

Thiamin

Headaches, irritability, insomnia, weakness

Vitamin B6

Dependency symptoms, toxic effect on nervous system, numb feet, unsteady gait

Eolate

May obscure pernicious anemia

Vitamin A

Blurred vision, fatigue, headaches, hair loss, dermal changes, liver damage

Vitamin D

Growth retardation, nausea, kidney damage, calcium deposits in soft tissues

Calcium

High blood calcium, kidney damage

Iron

Iron deposits in tissues which may lead to tissue damage, pigmentation of the skin

Zinc

Nausea, vomiting, abdominal cramps, diarrhea 
Long term use of certain vitamins even in doses as low as 5-10 times the RDA may lead to toxicity symptoms.

High doses of vitamins also interfere with the metabolism of certain drugs and, therefore, with the treatment and cure of certain diseases. Doses of nicotinic acid as high as 3 grams per day may cause release of the compound called histamine. As a consequence, flushing is a common side effect of nicotinic acid overconsumption. Taking an antihistamine may suppress the histamine release and reduce flushing. The acidity of nicotinic acid may cause stomach pain, especially in patients with peptic ulcer. High doses of vitamin C decrease the anticoagulant effect of the drug warfarin. In patients with iron storage diseases, vitamin $\mathrm{C}$ overconsumption may compound iron overload. Megadoses of vitamin C also increase excretion of amphetamines, while increasing absorption of aspirin. Large doses of vitamin B6 can increase the metabolism of the drug L-dopa to dopamine; thereby decreasing the effectiveness of L-dopa which is used to treat Parkinson's disease. Overconsumption of folate and vitamin B6 may also decrease the effectiveness of anticonvulsant medications.

Megadoses of minerals may be toxic and may interfere with the metabolism of other minerals. For example, overconsumption of phosphorous may enhance bone loss. Increased zinc intake generally reduces copper and iron absorption, while increased calcium intake may reduce the absorption of zinc.

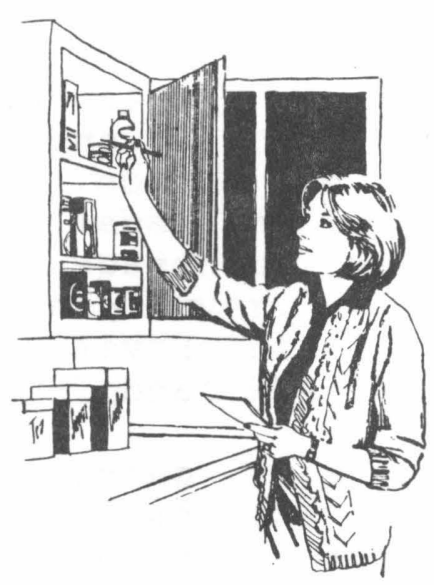

\section{SHOULD I TAKE VITAMIN \\ OR MINERAL SUPPLEMENTS?}

Before determining whether or not to take a supplement there are three things you should do:

\section{Determine the need for a supplement}

To do this, record everything you eat and drink for at least three days. Do not change your diet but be specific. To be sure you are consuming an adequate diet, compare your diet with the recommended number of servings from each of the food groups in the USDA daily food guide. Also, read extension publication number 348-906, "The Four Food Groups" which is available from your extension unit offices. 


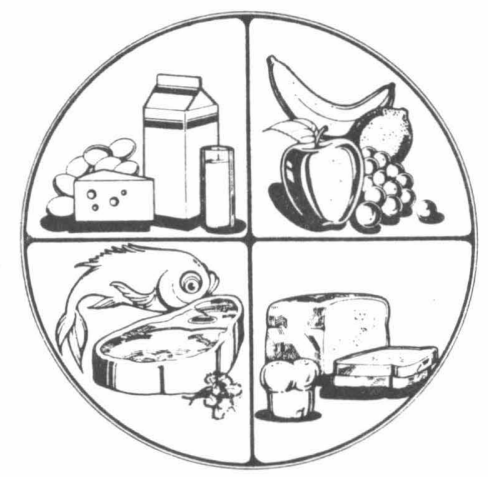

DAILY FOOD GUIDE

TABLE 2

DAILY FOOD GUIDE

$\begin{array}{cl}\text { Food Group } & \text { Minimum } \\ & \text { Recommended } \\ & \text { Number of } \\ & \text { Servings }\end{array}$
One Serving
is

Nutrients

supplied in

major quantities

Fruits and

Vegetables

Breads and

Cereals

(Whole grain)

(enriched)

Milk and

milk

products

Meat, meat alternates and beans
4

4

2

2
$1 / 2$ cup or small-medium size

1 slice or

$1 / 2$ cup cooked

1 cup or

$8 \mathrm{oz}$

2-3 oz, 2 eggs, 1 cup cooked beans vitamins $A$ and

$C$, and fiber

trace

minerals, B vitamins, and fiber

calcium, protein, riboflavin

protein, iron, zinc

Your extension home economist or a dietitian at a local hospital can give you a detailed analysis of your diet and he or she can compare your diet with the recommended dietary allowances (RDAs). The RDA for each nutrient is usually set by determining the average needs for the nutrient, increasing this average by $a$ value sufficient to meet the need of all healthy people (i.e. by adding a "safety factor"), and accounting for the efficiency of utilization of the nutrient in the body. Therefore the RDAs are liberal allowances higher than the needs of most individuals. 
If you find that your diet is nutritionally inadequate, the next thing to do is to improve your diet, making sure that you consume the recommended number of food group servings daily (as listed in the USDA daily food guide). Your physician can run a detailed analysis of a blood and urine sample to determine more accurately your deficiency status. For example, analysis of your hemoglobin and serum ferritin levels show whether you are anemic or not. These tests are expensive and are only performed if you have a nutrition-related health problem. Consumers should keep away from health promoters who use hair analysis to determine the need for a vitamin or mineral supplement. Deficiencies of these nutrients cannot be accurately determined using hair analysis. If you are deficient in a nutrient or you are unable to consume a nutritionally adequate diet, you may want to take a supplement. This decision should be discussed with your dietitian or physician.

\section{DETERMINE THE SAFETY OF YOUR SUPPLEMENT}

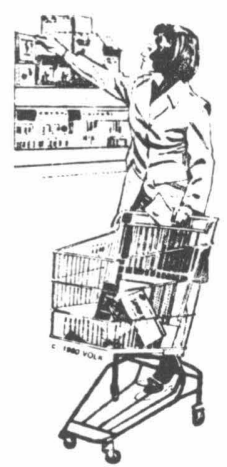

Read the label. The U.S. RDA (recommended daily allowance) used for food labelling is the maximum RDA of a specific nutrient for males, non-pregnant, non-lactating females, and children four years of age and over. These are standards set for comparing foods for nutrient content and not for nutrient intake. The U.S. RDAs are the highest RDA values for any age group.

The supplement that you decide to take should contain not more than $100 \%$ of the recommended dietary allowance for any nutrient and should consist of a wide array of nutrients. Table three lists the RDA for some vitamins and minerals for adults age 23 years and older.

Do not be misled by anyone trying to sell you "natural" vitamins. Vitamins are small but essential chemicals that your body cannot make and that must be supplied in the diet. Your body cannot tell whether the vitamin came from a rose hip or from the laboratory. Some of these vitamin tablets sold on the market also have other compounds that your body can synthesize and that are required only by some animals. For example, inositol, choline, and glutamic acid are not required by humans. 


TABLE 3
Recommended Dietary Allowance
(Daily nutrient intake allowance)

\section{IS THE SUPPLEMENT EXPENSIVE?}

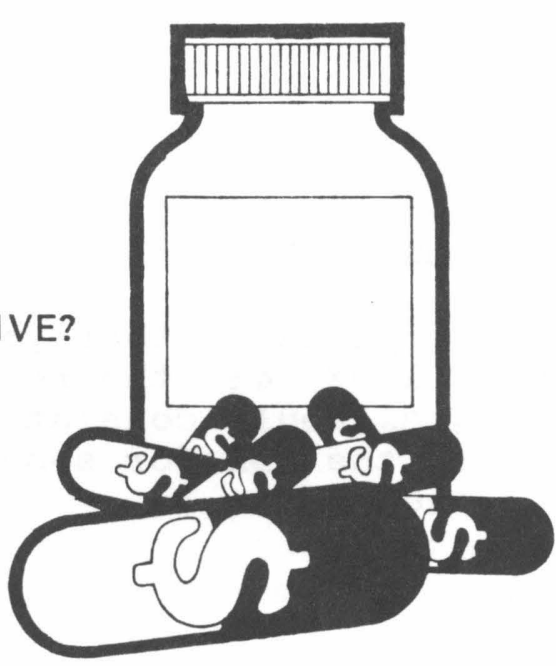

Vitamins and minerals are generally required in small amounts. You can obtain these nutrients more cheaply from food by consuming the recommended number of servings listed in the USDA daily food guide and by eating a varied diet. If you decide to supplement your diet, shop carefully for the supplement that contains the nutrient you want at the lowest possible price. Generic multivitamin preparations with iron are often your best buy. 


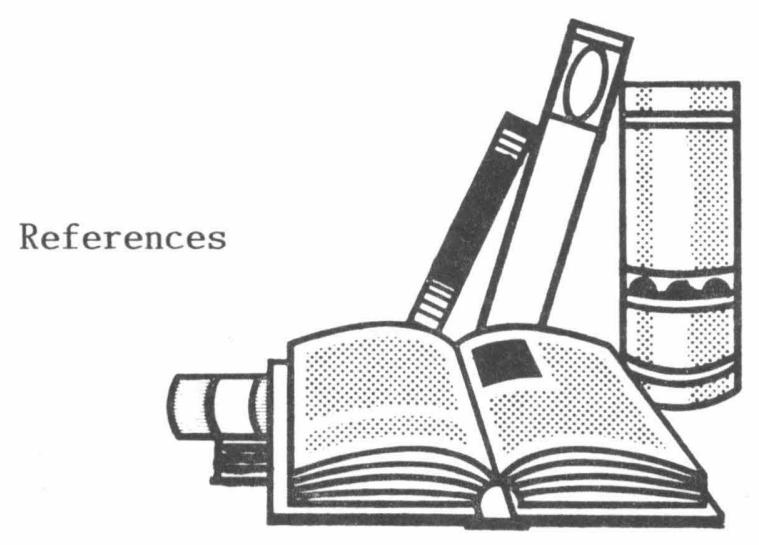

Books

Benowicz, Robert J. Vitamins and You. New York: Grosset and Dunlap, 1979 .

Herbert, Victor. Nutrition cultism Eacts and $\underline{\text { Eiction. }}$ Philadelphia: George E. Stickley, 1980.

Herbert, Victor, and Barret, stephen. Vitamin and Health Eoods: The Great American Hustle. Philadelphia: George F. Stickley, 1982 .

Marshall, Charles W. Vitamins and Minerals: Help or Harm. Philadelphia: George F. Stickley, 1983.

Journals

Alhadeff, Leslie et al. "Toxic Effects of Water-soluble Vitamins." Nutrition Reviews 42:2, (1984), 33-40.

"EDA Begins Move on OTC Vitamins and Minerals." American Pharmacy 19:50, (1979), 23-24.

Read, H. Marsha and Graney, S. Ann. "Food Supplement Use by the Elderly." Journal of American Dietetic Association 80, $(1982), 250-253$.

Schneider, L.C. and Nordlund, D.J. "Prevalence of Vitamin and Mineral Supplement Use in the Elderly." Journal of Eamily Practice 17:2, (1983), 243-247.

Virginia Cooperative Extension Service programs. activities. and employment opportunities are available to all people regardless of race.color. religion. sex. age national origin handicap or political affiliation. An equal opportunity/affirmative action employer.

Issued in furtherance of Cooperative Extension work. Acts of May 8 and June 30. 1914, and September 30, 1977, in cooperation with the U.S. Department of Agriculture. Mitchell R. Geasler. Director. Virginia Cooperative Extension Service and Vice Provost for Extension. Virginia Polytechnic Institute and State University. Blacksburg. Virginia 24061. Clinton V. Turner. Administrator. 1890 Extension Program. Virginia State University. Petersburg. Virginia 23803. 